\title{
Gradhiva
}

GRADHIV

Revue d'anthropologie et d'histoire des arts

3 | 2006

Du Far West au Louvre : le musée indien de George

Catlin

\section{Des Indiens de papier}

Entre réception royale et réception populaire

Paper Indians, between royal reception and popular reception

\section{Frédéric Maguet}

\section{CpenEdition}

\section{Journals}

Édition électronique

URL : http://journals.openedition.org/gradhiva/225

DOI : $10.4000 /$ gradhiva.225

ISSN : 1760-849X

Éditeur

Musée du quai Branly Jacques Chirac

Édition imprimée

Date de publication : 15 juin 2006

Pagination : 89-103

ISBN : 2-915133-26-3

ISSN : 0764-8928

\section{Référence électronique}

Frédéric Maguet, «Des Indiens de papier», Gradhiva [En ligne], 3 | 2006, mis en ligne le 15 mai 2009,

consulté le 01 mai 2019. URL : http://journals.openedition.org/gradhiva/225 ; DOI : 10.4000/ gradhiva.225

Ce document a été généré automatiquement le 1 mai 2019.

(c) musée du quai Branly 


\title{
Des Indiens de papier
}

Entre réception royale et réception populaire

Paper Indians, between royal reception and popular reception

\author{
Frédéric Maguet
}

1 «Deux sauvages à Paris, n'est-ce pas assez rare pour que nous en parlions? » interroge L'Illustration du 15 février 1845. Il s'agit pour l'heure de « Deux Indiens sauvages des forêts vierges du Brésil » ramenés par un explorateur français pour être étudiés par l'Académie des sciences. Nul ne s'émeut de les voir traités comme des spécimens, qu'il convient d'isoler tout au long du voyage pour leur éviter des contacts qui pourraient les pervertir, car il s'agit de les présenter aux savants « sauvages comme ils l'étaient dans leurs forêts ». Les gravures qui accompagnent l'article, les fameuses gravures en bois de fil qui ont fait le succès des débuts de L'Illustration - le journal en est à sa troisième année de parution - les présentent en buste, paradoxalement vêtus à l'européenne, et le texte précise qu'ils acceptèrent difficilement ces vêtements qu'on voulut leur donner pour le débarquement. La présence de sauvages à Paris est un fait assez rare pour être remarqué, commenté... et surtout représenté : le XIX ${ }^{\mathrm{e}}$ siècle est l'ère du développement de l'image à la portée de tous, c'est l'époque des grandes innovations techniques qui, en permettant un accroissement fulgurant de la production imagière, favoriseront l'émergence d'une encyclopédie visuelle partagée. L'intérêt porté aux peuples, les plus lointains comme les plus proches, s'inscrit directement dans ce mouvement de systématisation de l'imaginaire commun. Les termes en sont déjà fixés de longue date, notamment par les illustrations de récits de voyage, mais la nouveauté réside dans l'interpénétration entre une typologie de base, fondée sur des attributs, et des saisies documentaires directes qui renouvellent les répertoires iconographiques, sans pour autant subvertir fondamentalement les types auxquels elles s'appliquent. 


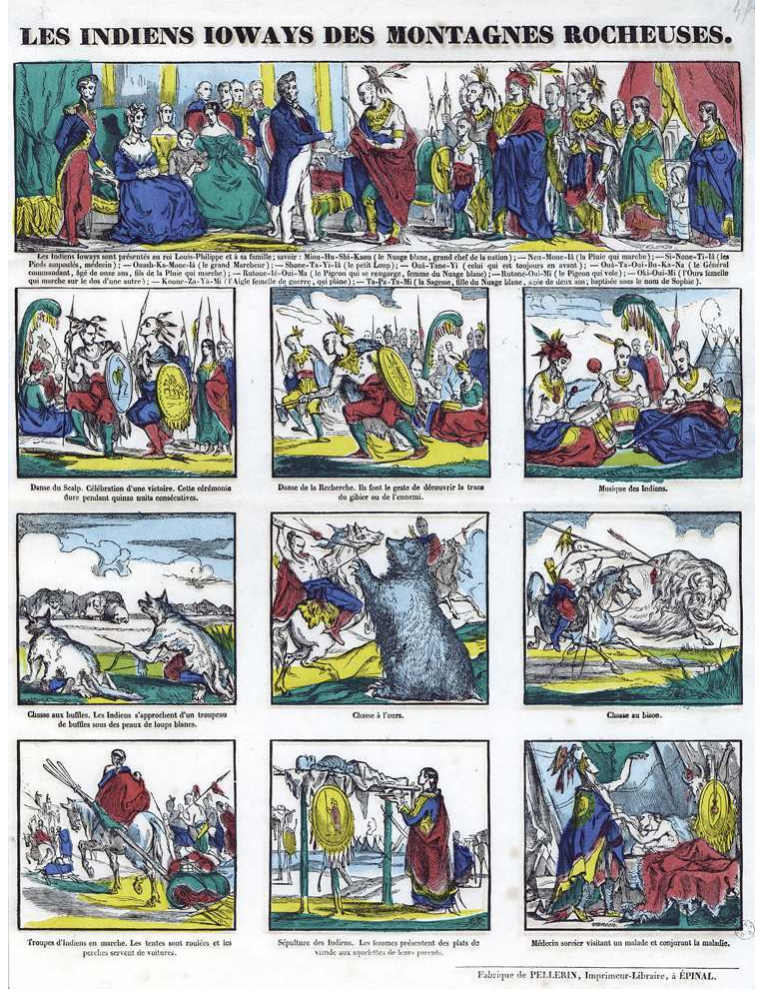

Iconothèque, musée des Civilisations d'Europe et de la Méditerrannée.

Les attributs de l'Indien sont fixés très tôt : dès le XVI $\mathrm{e}^{\mathrm{e}}$ siècle, les plumes, la nudité souvent tempérée par un pagne, les peintures corporelles et l'arc constituent les éléments d'un système représentatif fortement ancré. On les trouve au début du XIXe siècle chez Grasset Saint-Sauveur, dans ses Voyages pittoresques. On les trouve un peu plus tard dans l'estampe populaire, par exemple sur une image éditée à Caen entre 1831 et 1835 dans une composition à la gloire de "Notre Dame de Grâce, étoile de la mer, protectrice des matelots et des passagers ». On les trouve également dans les années 1840, quand l'imagerie parisienne entreprend d'illustrer les romans à la mode, notamment Atala, ou de peindre l'histoire de Cortés ou celle de Colomb. Ces images d'Indiens, tout à fait fantaisistes pour notre regard moderne, ressortissent d'une construction allégorique: elles représentent l'Amérique dans la série des Quatre parties du monde et sont, par ce biais, mobilisées non seulement dans la peinture et l'estampe mais aussi dans les spectacles. Divertissements princiers et cortèges populaires mettent en scène des "Incas ", des «Indiens ", des "Sauvages américains », les dénominations importent peu, qui arborent fièrement des parures de plumes, car tel est l'attribut dominant qui les distingue des «Romains » ou des « Turcs »; la nudité étant exclue des spectacles vivants, tout le monde est en général vêtu d'une tunique et chaussé de sandales. Tel est l'Indien classique, l'Indien allégorique dont les traits sont très fortement mêlés à ceux de l'homme sauvage dont il emprunte parfois les attributs. En 1828, Pellerin, l'imagier d'Épinal, édite une estampe intitulée Sauvages de l'Amérique qui se compose de quatorze figures identiques sur le modèle des planches de soldats à découper - représentant un homme armé d'une massue et d'une fronde (les armes classiques de l'homme sauvage), mais aussi, bizarrement, d'un glaive. Il est vêtu d'un pagne agrémenté d'une ceinture de plumes, il 
porte collier et bracelets, et est coiffé de l'inévitable couronne en plumes d'autruche. C'est la structure de cette mise en représentation, qui s'appuie à la fois sur l'estampe et sur le spectacle, qui lui confère une grande stabilité ; mais c'est elle également qui va lui permettre de se transformer en acceptant des modifications « réalistes» des attributs, dès lors que les spectacles mettront en scène des Indiens réels, et non plus des Français déguisés. Il y aura alors, au sein de cette structure représentative, négociation entre des traits concurrents tendant à renouveler le stéréotype par un double mouvement de complexification puis de simplification. Mais à aucun moment le principe du stéréotype n'est en crise bien que l'on puisse avancer, comme nous le verrons, que ce double mouvement correspond à une mutation radicale dans le mode même de construction du stéréotype, au passage d'un régime constitutif à un autre. Au bout du processus, le résultat final est que, en 1900, l'allégorie de l'Amérique dans les Quatre parties du monde est figurée par un (ou une) Sioux, de même que les déguisements - pour les spectacles de cirque comme pour les jeux d'enfants - emprunteront à l'iconographie désormais fixée des Indiens des Plaines.

3 De ce point de vue, le passage à Paris du musée itinérant de George Catlin est tout à fait décisif car il se situe à un moment où les deux facteurs constitutifs de la structure représentative, le spectacle et l'image, connaissent une mutation importante. Il est d'autant plus marquant qu'il est accompagné d'une «exhibition» exécutée par les Indiens iowas dans laquelle "tout le monde a reconnu du premier coup d'œil des sauvages authentiques, et non des sauvages de la banlieue ", ainsi que l'annonce PitreChevalier dans Le Musée des familles. Les visites d'Indiens sont rares, certes, mais elles ne constituent pas une nouveauté : depuis les premiers Indiens ramenés par Christophe Colomb à la cour d'Espagne, chaque siècle a connu son lot de visites d'hommes et de femmes, parfois invités et parfois captifs ; il n'est que de citer la rencontre de Montaigne avec les Indiens du Brésil amenés à Rouen en 1550. Trois traits caractérisent les visites du $\mathrm{XIX}^{\mathrm{e}}$ siècle : d'une part, on note une représentation croissante des peuples de l'Amérique du Nord; d'autre part, on relève l'importance accordée à l'argument scientifique dans le discours public (les Iowas, comme les deux Indiens des forêts vierges du Brésil, sont examinés par les savants de l'Académie, et ce fait est connu). Enfin, on ne peut qu'être attentif à la publicité faite à ces visites, qui prennent non seulement la forme classique de l'ambassade, mais celle, nouvelle et mouvante, du spectacle populaire. Cependant, les réceptions royales perdurent: de même que les six Osages débarqués au Havre en 1827 avaient été reçus par Charles x, les Iowas sont reçus le 21 avril 1845, aux Tuileries, par Louis-Philippe et sa famille, point d'orgue d'une série de réceptions qui leur fait rencontrer des personnalités comme l'ambassadeur des États-Unis et le préfet de la Seine. Le numéro de L'Illustration du 26 avril, leur consacrant un premier article, note : «Après avoir fréquenté les rois, les princes, les préfets et les doctes, il est probable que ces rudes enfants des montagnes Rocheuses descendront dans le populaire, et se montreront incessamment au premier venu moyennant une honnête rétribution, dans la salle Musard, au boulevard du temple, ou dans quelque vaudeville pantomime, tragédie, opéracomique, préparés tout exprès. » Le chroniqueur de L'Illustration est méfiant, il s'attend visiblement à une escroquerie ou à un spectacle plus ou moins dégradant; sans doute aussi a-t-il en mémoire le sort lamentable des Osages de 1827 qui, après avoir parcouru une partie de l'Europe, furent abandonnés par leur impresario dans le plus complet dénuement et ne purent rentrer chez eux que grâce à une souscription charitable. Mais il réserve son jugement : «[...] remettant une description plus complète de leurs personnes 
et de leurs mérites au temps où il nous aura été permis de les approcher de plus près, et de savoir positivement à qui nous avons affaire. »Ce premier article est illustré par un bois assez médiocre représentant un portrait de groupe des Iowas montrant le chef Nuage-Blanc au centre, entouré des guerriers, avec les femmes à l'arrière-plan; cette image est la première à avoir été diffusée massivement auprès du public français ${ }^{1}$. Le bois, non signé, est vraisemblablement de la main d'Henri-Désiré Porret, graveur qui a travaillé à l'illustration de livres, notamment en collaboration avec Tony Johannot ${ }^{2}$.

Chasse aux buffles par une tribu, xylographie, L'Illustration, 7 juin 1845, dessiné par Pharamond Blanchard d'après George Catlin.

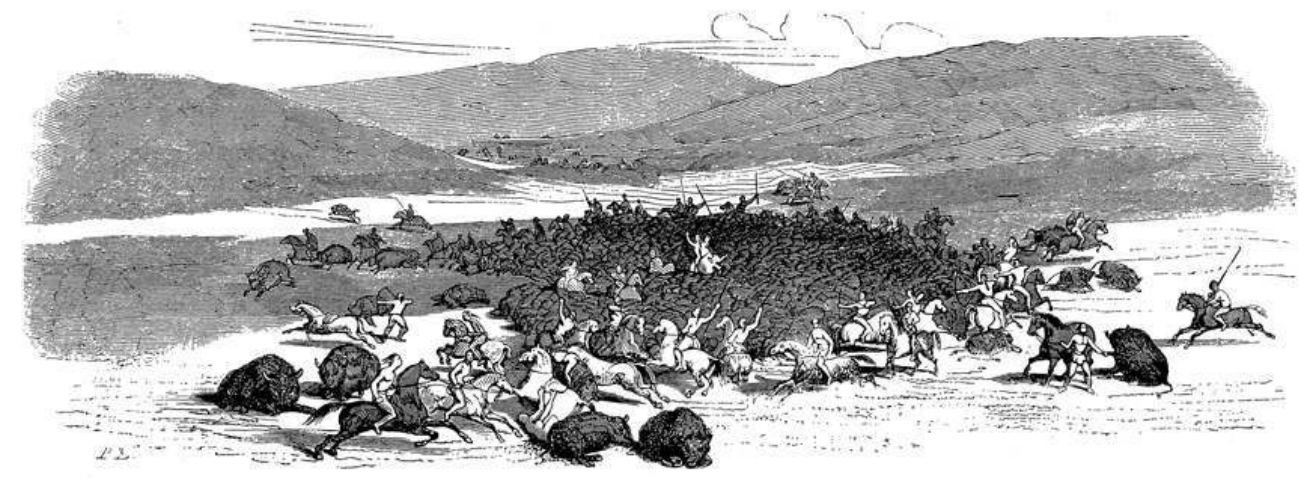

collection particulière

4 L'Illustration a raison dans le principe, les Iowas, comme on le sait, vont se produire salle Valentino; mais, comme on le sait également, le spectacle n'aura rien à voir avec un quelconque vaudeville. La notice éditée pour l'occasion comporte les «noms des douze Indiens Ioways ", différents textes du Bureau des Affaires indiennes vantant leurs mérites, des descriptions de leurs coutumes et de leurs manières de vivre, un compte rendu de leur visite à Louis-Philippe, ainsi que le programme du spectacle; en somme : un ensemble de textes disparates, visiblement rassemblés à la hâte. Cet aspect de fourre-tout est largement compensé par la richesse de contenu, fort bienvenue pour un public lettré qui, jusque-là, ne connaissait les Indiens que par les œuvres de Cooper et de Chateaubriand, et davantage encore pour un public un peu plus large qui n'avait en tête que les stéréotypes véhiculés par l'imagerie. La notice est illustrée de neuf gravures signées par Porret, toujours d'un style un peu fruste, qui reproduisent des tableaux de Catlin. Quatre sont des portraits en pied, mais on trouve aussi la célèbre chasse au bison avec le cavalier tirant à l'arc, le joueur de balle, ainsi qu'un groupe de wigwams ; les deux dernières gravures représentent des numéros du spectacle : la danse de l'ours et la danse du scalp. La plupart des titres renvoient à une page du texte. C'est là une des mutations majeures de l'image populaire dans les années 1840-1850 : cette interrelation entre un texte informatif et une illustration, classique pour l'édition de livres, est tout à fait nouvelle pour ce type d'ouvrages consacrés à une actualité immédiate. La formule adoptée par la notice de la salle Valentino est à vrai dire assez proche de celle de L'llustration: un texte narratif fait référence à une figure, qui à son tour renvoie au passage qu'elle illustre.

5 Concernant Catlin, L'Illustration s'engage dans une série d'articles qui nous permet d'évaluer de manière très précise, presque semaine après semaine, la faveur dont jouit auprès du public le spectacle des Iowas. Le parti pris est celui qui est suivi par le journal pour d'autres thèmes dont l'intérêt est jugé évident, tels les « Mœurs et coutumes de 
Basse-Bretagne " ou les reportages sur le Maroc et l'Algérie: on ne propose plus de chronique, mais des textes généraux à vocation didactique. Après le premier article du 26 avril, qui, comme on l'a vu, restait dans l'expectative, les numéros datés du 24 mai, du 7 juin et du 21 juin 1845 témoignent d'une adhésion sans réserve à l'entreprise. Ils offrent de longs textes de Catlin traduits assez librement par Xavier Eyma et qui consistent en récits de voyages, tant sous la forme de souvenirs que sous celle de descriptions de coutumes indiennes, ainsi qu'en un manifeste de la cause indienne. Catlin se veut militant, son projet étant, par rapport aux Indiens, de « détruire les absurdes préjugés qu'on nourrit contre eux, pour les défendre contre ce paradoxe que des gens qui ne les connaissent pas ont mis en circulation, à savoir : qu'ils sont une anomalie de la nature ». Il trouvera dans L'Illustration une excellente tribune pour ses idées. Aucune référence n'est faite au spectacle, comme si l'événement était trop connu pour qu'il soit utile de le rappeler ; cette impression sera confirmée par L'Almanach de L'Illustration pour 1846, qui juge que les Indiens iowas ont été « les rois de la mode, les lions de la saison [...] ce fut même mieux que de la curiosité, ce fut un intérêt courtois, empressé, pour les chefs américains ; on se passionna en quelque sorte pour leurs jeux bizarres, pour leurs mœurs lointaines, pour leur étrange costume ». Nous sommes bien loin du sort de spécimen vivant réservé quelques mois plus tôt aux deux Indiens du Brésil. Les illustrations sont à la hauteur des textes: les trois numéros publient en tout vingt-trois gravures, et il ne s'agit plus du travail un peu rapide de Porret. On peut identifier deux dessinateurs qui ont interprété des peintures de Catlin et qui sont, dans leur genre, des figures de tout premier plan. Plusieurs planches, notamment Chasse aux buffles par une tribu et La Danse du scalp sont monogrammées « PB », lisons Pharamond Blanchard ${ }^{3}$. D'autres gravures, telles que Le Chien-Noir et Le Penseur, sont dues à Eustache Lorsay, écrivain et peintre reconnu pour ses portraits et ses tableaux historiques. Davantage qu'aux Iowas, les trois numéros sont consacrés au travail de Catlin dont ils constituent un prolongement efficace pour la défense de la cause indienne. 

Catlin.

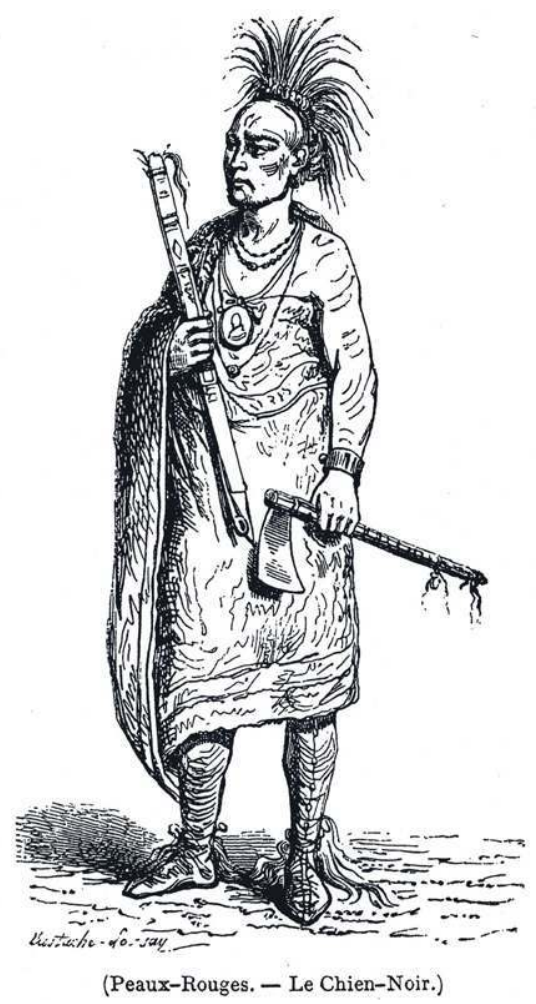

collection particulière

Mais le 2 août, à l'occasion des obsèques de la femme du Petit-Loup, L'Illustration publie un nouvel article au ton beaucoup plus personnel puisqu'il traite essentiellement de l'histoire de la défunte : « Lorsque l'Indienne Sawk-O-Ki-Oui-Mi, plus connue sous le nom de Madame Petit-Loup, eut été ensevelie au cimetière Montmartre, les Français qui assistaient à cette cérémonie funéraire promirent au Petit-Loup de faire élever un tombeau à la mémoire de sa femme... » L'article est illustré par une gravure représentant le projet du tombeau ainsi qu'un fac-similé du dessin exécuté par le fils de la Pluie-quimarche représentant l'histoire de la vie du Petit-Loup. Le tombeau ne sera jamais terminé, seuls le bas-relief qui devait l'orner et le buste d'O-Ki-Oui-mi seront exécutés par Auguste Préault. La fin de l'article est essentiellement constituée d'une reprise du texte de George Sand paru dans Le Diable à Paris racontant l'histoire du Petit-Loup et de sa femme ${ }^{4}$. Cet événement, que L'Almanach qualifiera de « deuil général », semble marquer le moment de plus forte sympathie des Parisiens à l'égard des Iowas; une fois ceux-ci rentrés aux États-Unis, l'intérêt s'estompe aussi brutalement qu'il avait débuté. Les Iowas n'ont séduit un public versatile, qui a dépassé la simple curiosité, que pour un temps très court. Le 23 août, L'Illustration publie une gravure de qualité assez médiocre, très sombre, intitulée "Indiens O-jib-ha-Way nouvellement arrivés à Paris»; aucun texte n'accompagne ce portrait de groupe, aucune explication sur le fait qu'une troupe d'Ojibwas est arrivée de Londres pour remplacer les Iowas salle Valentino puis au Louvre, aucun nom n'est cité. L'Illustration ne présentera plus d'articles sur les Indiens avant le départ de Catlin, concentrant son intérêt sur les événements d'Algérie. C'est L'Almanach de 1846 qui donnera une explication, très brutale, à ce retournement de situation: «L'accueil qu'avaient reçu les Ioways tenta une autre tribu; plusieurs O-jib-be-was 
arrivèrent à Paris. Mais ce n'est pas le tout d'être Indiens, il faut encore arriver à temps, et les O-jib-be-was trouvèrent l'enthousiasme pour l'Amérique du Nord singulièrement refroidi ; on alla même, pour se dispenser de les visiter, jusqu'à mettre en doute leur identité, et quelques sceptiques prétendirent que les O-jib-be-was étaient des Indiens de la Basse-Bretagne. » L'Almanach reprend deux portraits publiés dans le journal, mais sans légendes. L'Illustration est le seul vecteur de grande diffusion qui popularise le travail de Catlin au moment même où il se produit à Paris; rien dans Le Magasin pittoresque, un seul article, non illustré, dans Le Musée des familles. On notera d'autre part que, pour ces journaux, il s'agit essentiellement d'un événement d'actualité : on ne trouve aucun article sur les Indiens ou sur Catlin au cours des années suivantes.

7 Pour autant, l'événement n'est pas passé inaperçu, et il a servi de support à plusieurs débats d'opinion concernant les rapports entre la « civilisation » et les « sauvages». Un premier point concerne le caractère supposé immoral des exhibitions; bien avant les "zoos humains" de la fin du siècle, mais après la Vénus hottentote, certains s'interrogent, tel Pitre-Chevalier dans Le Musée des familles: "Ces exploitations et ces exhibitions d'hommes, quels qu'ils soient (et il y a là une femme chrétienne) ont quelque chose de révoltant au milieu d'une civilisation qui repose sur la liberté. On dira que ces exercices et cette publicité font la joie des sauvages, qui aiment par-dessus tout se montrer et à poser devant la foule. Nous répondrons que nous doutons qu'ils aiment à jouer la comédie, à danser à l'heure sur l'ordre d'un cornac, et à manger des blanquettes de veau dans un hôtel garni. » Un second point concerne la question de la responsabilité des nations civilisées dans la destruction - jugée par ailleurs inévitable - du monde sauvage. Ce thème est développé longuement dans L'Illustration, par Catlin lui-même : « Si toute cette race d'Indiens était venue à disparaître du monde par les ravages de quelque épidémie, il eût paru naturel, n'est-ce pas, qu'on s'apitoyât sur leur sort? Eh bien! Ne leur devons-nous pas aussi quelque sympathie, quand il est certain que c'est nous-mêmes qui les tuons, que ce sont nos propres mains qui leur donnent la mort! [...] Il faut reconnaître aussi qu'il n'est pas possible à un gouvernement qui poursuit un projet bien arrêté, ni à des marchands avides d'argent, de se frotter à ces natures crédules autrement que pour leur faire endurer de cruelles injustices. Ce n'est donc pas de la part du gouvernement ni de la part des trafiquants qu'il faut s'attendre à aucune pitié ; mais moi, humble biographe, je reviens de ce pays les mains vides et la conscience pure, je puis leur rendre hautement justice, à ces pauvres Indiens, et c'est ce que j'ai tâché de faire. » $\mathrm{Ce}$ thème, fort délicat, sera repris par le caricaturiste Cham, dans trois planches satiriques assez brutales intitulées Épisodes de l'histoire d'une nation sauvage, ou les bienfaits de la civilisation $^{5}$, mettant en scène le Nuage-Blanc de retour au pays. Traité sur le mode burlesque, elles présentent le chef indien sous un jour peu favorable : vêtu d'un uniforme occidental, coiffé de l'immense bonnet à poils que l'on retrouve dans de nombreuses caricatures de Cham, il cherche à asseoir son pouvoir en essayant de convaincre les Iowas d'adopter les coutumes et les lois européennes, et en impressionnant les dignitaires de la tribu avec des objets rapportés d'Europe. La chute, prévisible, se termine par une catastrophe : « Le jour même, les allumettes chimiques ayant incendié quinze villages, les I-o-ways commencent à réfléchir aux bienfaits de la civilisation. » 
La giraffe (sic) visitée par les sauvages indiens au jardin du roi à Paris, xylographie coloriée au pochoir sur papier vergé, Paris, Tautin, 1828.

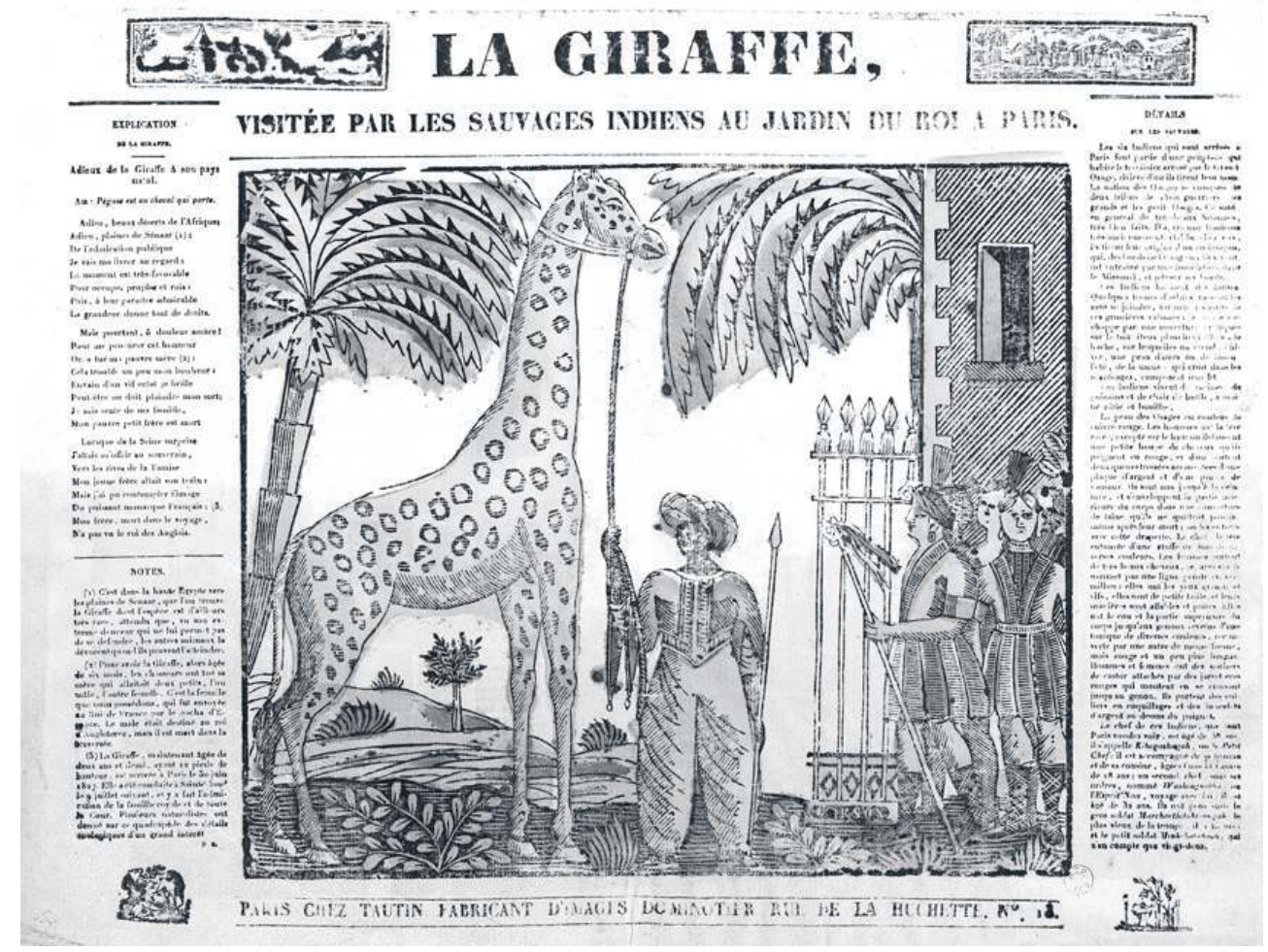

Iconothèque, musée des Civilisations d'Europe et de la Méditerrannée.

On peut se demander pour qui étaient exposés de tels débats - sous forme sérieuse ou satirique - et pour qui se produisaient les Iowas. Quels rapports, en d'autres termes, peuton essayer de tracer entre l'événement parisien et ses prolongements journalistiques d'une part, le stéréotype commun - populaire - de l'Indien d'autre part? Le spectacle de la salle Valentino a eu un impact immédiat, mais limité au public de ce type de salles et aux lecteurs des journaux illustrés, c'est-à-dire deux populations sociologiquement proches. Si l'on connaît le tirage de L'Illustration, on a en revanche peu d'éléments sur le nombre de spectateurs qui ont pu personnellement assister au spectacle. Il est tout de même possible de fixer un ordre de grandeur. Dans la lignée des Concerts-Musard, la salle Saint-Honoré, qui prend en 1841 le nom de Bal Valentino, s'étend sur 600 mètres carrés ; elle est une des sept ou huit grandes salles de bal capables d'accueillir 2000 danseurs. En 1845 , ces salles qui, dans la décennie précédente avaient réussi à conquérir un public élégant, sont sur le déclin : François Gasnault indique que « la clientèle des bals d'hiver ne se recrute pas au-delà des premières couches de la petite bourgeoisie : artisans, employés, vendeurs des magasins de nouveauté »; ce n'est pas le public populaire des guinguettes mais ce n'est pas non plus la grande bourgeoisie et l'aristocratie. Quant à la disposition physique des lieux, elle peut être décrite de manière générique : "Autour de la salle de danse, plus ou moins illuminée, plus ou moins décorée, enguirlandée, peinturlurée, règne une galerie que supportent des poteaux déguisés en colonnes. [...] La buvette est établie au premier étage et les consommateurs se tiennent dans la galerie, d'où ils aperçoivent l'ensemble des danses.» (cité in Gasnault 1986: 131). Pour survivre, ces salles sont obligées de se diversifier; classiquement salles de bal et de concerts (alternativement de musique « sérieuse » et de musique « légère »), elles accueillent des cours de danse, mais 
aussi des banquets et des spectacles ${ }^{6}$. Le tarif d'entrée pour les soirées de bal est en moyenne de cinq francs; on ne connaît pas celui des spectacles. Les Iowas ont donc accompli une soixantaine de représentations dans une salle de 600 mètres carrés; compte tenu de la disposition des lieux, qui incluent une galerie en surplomb, on peut estimer que des conditions correctes pouvaient être garanties pour un millier de spectateurs. On ne peut en revanche savoir si le spectacle fit salle comble; il faut en douter puisque Catlin fut obligé de se replier sur un autre lieu, plus prestigieux mais plus petit : la salle des états du Louvre, qu'il obtint par une autorisation du roi accordée grâce à l'intervention de Karl Girardet. Toutes ces données inclinent à penser que le spectacle de la salle Valentino a été vu par un public qu'on peut évaluer entre 30000 et 50000 personnes, public composé de membres de la petite bourgeoisie évoqués plus haut, mais aussi de curieux attirés par ce spectacle en particulier, au premier rang desquels, bien sûr, les artistes romantiques de la jeune génération qui jouèrent, mais à retardement, un rôle de relais d'opinion.

9 Sur le plan strictement iconographique, très peu d'images des Iowas sont produites durant leur séjour à Paris : les neuf bois de Porret qui illustrent la notice du spectacle, et une lithographie de Buchot figurant sur une partition d'A. Ropicquet intitulée Les Indiens à Paris, c'est-à-dire par définition des planches à diffusion restreinte. Bien sûr, les artistes s'intéressent aux Iowas: Delacroix, qui exécute une série de dessins, Karl Girardet qui peint, à la demande du roi, une toile intitulée Louis-Philippe assistant dans un salon des Tuileries à la danse d'Indiens Hovas. 21 avril 1845, ou encore Théodore Gudin, qui utilise les figures des Indiens pour une grande composition intitulée Jacques Cartier découvre le SaintLaurent. Si l'on ajoute sept feuilles d'étude attribuées à Auguste Raffet ${ }^{7}$, on a là la totalité de l'influence directe du spectacle des Iowas sur les peintres. Les dessins et les peintures ne sont pas reproduits, quant aux gravures, elles sont d'une part trop précises, trop documentaires, pour proposer une vision unifiée de l'Indien qui puisse rivaliser avec les classiques "sauvages de l'Amérique", et en même temps trop confidentielles pour pénétrer profondément les milieux populaires, cela malgré le succès incontestable que connaît L'Illustration.

10 Aussi, pour important à maints égards que fut l'événement, il ne toucha pas directement le public populaire. On le remarque aisément au fait que la grande absente, parmi les relais possibles vers un large public, c'est l'image à la feuille, et particulièrement l'imagerie parisienne de la rue Saint-Jacques ${ }^{8}$. À cet égard, le sort réservé à l'image des Iowas en 1845 (ne parlons même pas des Ojibwas) est incomparablement moins favorable que celui qui avait été octroyé à l'image des Osages en 1827. Jean, l'un des éditeurs les plus connus et les plus anciens de la rue Saint-Jacques, avait publié une planche intitulée Mours et usages des Indiens de la tribu des Osages, arrivés en France en 1827; d'autres estampes avaient circulé, par exemple deux eaux-fortes anonymes, sous les titres de Prince Osage et Roi des Osages descendu à l'Hotel de la Terrasse rue de Rivoli. Même Engelmann, qui expérimentait tout juste la lithographie en couleur, avait édité La Curiosité civilisée punie par la barbarie. Aventure de la rue de Rivoli, où l'on voyait un Osage jeter le contenu d'un pot de chambre, depuis son balcon, sur un public trop curieux. Enfin, on trouve chez Tautin une image très fruste, à la limite du canard, intitulée La giraffe (sic) visitée par les sauvages indiens au jardin du roi à Paris, illustrant une rencontre (réelle ou fictive) entre les deux attractions de la saison, c'est-à-dire les Osages et la girafe Zafara, offerte à Charles X par le pacha d'égypte. L'imagerie des centres provinciaux n'était d'ailleurs pas en reste puisque les portraits des Osages ornaient dès 1828 des calendriers de Rouen, ville où leur passage avait constitué un événement considérable. Et l'empreinte avait été suffisamment 
profonde pour que leurs traits distinctifs soient réutilisés par la suite. Ainsi, si l'histoire d'Atala était illustrée par l'imagerie parisienne dans un style qui alliait l'influence du néoclassicisme de Girodet et celle du stéréotype du sauvage indien ${ }^{9}$, il n'en était pas de même à Metz où Dembour édite, entre 1835 et 1840, une image intitulée Histoire religieuse d'Attala ou de la fille du désert, sur laquelle Chactas est clairement dépeint sous les traits d'un Osage. Deux éléments sont venus s'agréger au vocabulaire du stéréotype initial parce qu'ils étaient d'emblée compatibles avec son mode de construction: la coiffure et l'armement. La coiffure des Osages tranche radicalement avec la couronne de plumes d'autruche des «Incas »; pratiquement identique à celle des Iowas, elle est d'ailleurs fort bien décrite par Catlin lui-même dans l'article de L'Illustration du 21 juin 1845 : « Les Y-ovays, les Osages, les Pawnees et les Sacs et Foxes ont seuls l'habitude de se raser la tête. [...] Ils ne conservent de leur chevelure, coupée aussi ras que possible, qu'une touffe au sommet de la tête, de la grosseur du poing, et de deux pouces de haut, au milieu de laquelle ils plantent une belle crinière de cheval ou de daim, mélangée de plumes d'aigle. " Magnifiquement représentée sur un buste d'Indien sculpté par Jean-Pierre Dantan $^{10}$, cette coiffure tendra peu à peu à supplanter la couronne de plumes dans l'image populaire. Elle dominera jusqu'en 1889 , date à partir de laquelle elle cohabitera avec les cheveux longs et les parures de plumes des Sioux de Buffalo Bill. L'arme des Osages, c'est le tomahawk, en particulier celui très orné du chef Kishagashuguk tel qu'il est représenté par Louis-Léopold Boilly. On le trouvait certes déjà dans les représentations classiques, où il était figuré comme une simple hache ${ }^{11}$, mais de manière très discrète, et de toute façon jamais sur les images à la feuille. À partir de 1827, le tomahawk fera partie, au même titre que l'arc et les plumes, des traits qui désignent immédiatement l'Indien.

Cortés renverse les idoles des Mexicains, taille-douce, Paris, Bulla, 1845.

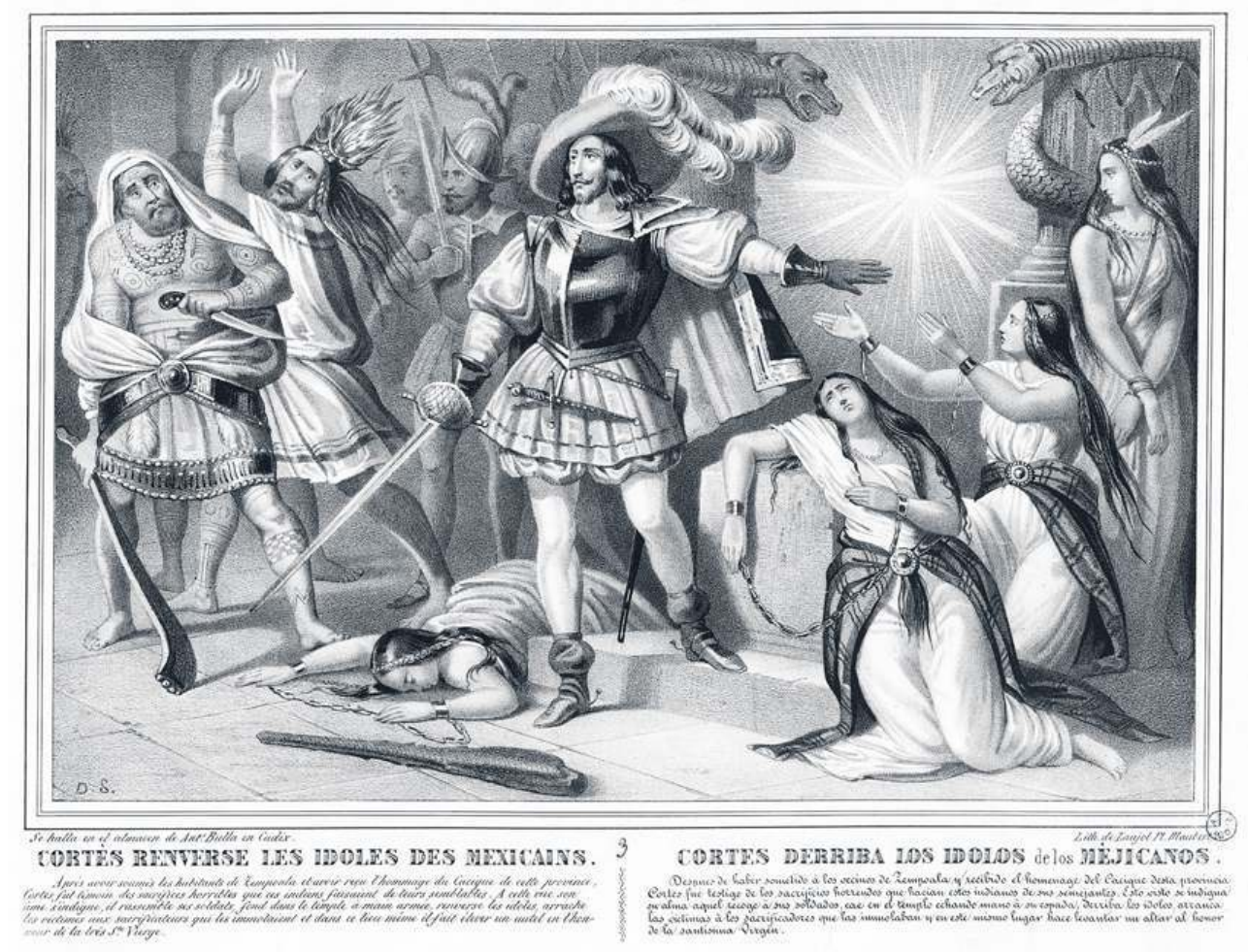

Iconothèque, musée des Civilisations d'Europe et de la Méditerrannée. 
11 On aurait pu s'attendre à ce que cet intérêt des imagiers de 1827 se répète en 1845 ; or, aucun des grands éditeurs de l'imagerie parisienne ne s'intéresse aux Iowas. Il semble que, paradoxalement, l'effort documentaire développé par George Catlin, à travers la présentation de son musée itinérant, ait eu un effet de brouillage vis-à-vis d'un public à la recherche de repères visuels simples. Les journaux illustrés - alors en émergence - et l'imagerie populaire ne fonctionnent pas selon les mêmes critères, et ce qui garantit la réussite dans un domaine peut constituer un handicap sérieux dans l'autre. Les grands éditeurs de l'époque, Bulla, Cereghetti, Turgis, Codoni, ne boudent pas les Indiens, mais ils préfèrent le stéréotype classique. En 1845, alors que les Iowas sont à Paris, la mode est aux représentations historiques, notamment celle de Cortés. On trouve le thème illustré au Salon par Pharamond Blanchard avec le tableau Fernand Cortez allant de la Vera-Cruz à Mexico. On le trouve surtout dans l'imagerie; l'histoire du conquérant est publiée en séries, généralement de quatre planches, qui en retracent les faits les plus remarquables ${ }^{12}$ . Lorsque Turgis représente Montezuma prisonnier de Fernand Cortés, le roi mexicain - barbu - porte une toge, une cape et des sandales, et il est coiffé d'une magnifique couronne de plumes. Ces stéréotypes des images populaires ne sont pas des systèmes simples, et surtout pas des systèmes clos, ils engagent tout un réseau de correspondances fondées sur des rapprochements et des oppositions souvent étonnantes pour l'œil contemporain. Ainsi, il peut paraître étrange que l'« homme de Californie » et « la femme du Pérou » de Grasset Saint-Sauveur soient coiffés d'un turban, certes surmonté de plumes, mais de forme tout à fait arabisante. De même, on peut s'étonner que chez Bulla, en 1845, Cortez renverse les idoles des Mexicains mette en scène un prêtre sacrificateur clairement déguisé en rabbin. Ces transferts de motifs visuels ne sont pas gratuits, ils ont leur pendant dans l'ordre du discours et relèvent même parfois d'un discours tout à fait construit. Ainsi, l'assimilation des Indiens et des juifs correspond à deux théories encore très en vogue à l'époque: la théorie classique voulant que les Indiens descendent des tribus perdues d'Israël et une version plus moderne professant que les juifs, chassés par les chrétiens, se seraient mêlés aux populations indiennes natives. Ces opinions étaient alors tellement diffusées qu'on retrouve la seconde sous la plume même de Catlin, très fin observateur des mœurs indiennes mais en même temps homme de son temps. On lit ainsi, dans le numéro de L'Illustration daté du 21 juin 1845 : "Certaines de leurs coutumes sont bien positivement calquées sur les traditions juives ; et, à cause de cela, je suis porté à croire que des débris des anciennes tribus israélites, dispersées par les chrétiens sur tant de points de la terre à tant de reprises et à des époques si différentes, auront trouvé à se réfugier dans l'Amérique du Nord, où elles se seront mêlées et entremariées avec les Indiens primitifs. [...] Ainsi, la fameuse cérémonie du mystère des quatre jours chez les Mandans, le privilège octroyé à quelques chefs de porter des cornes sur la tête, enfin, la plupart de leurs traditions religieuses, telle que celle du déluge, etc., font foi de ces assertions. "

12 Dans la seconde moitié du siècle, en réalité, le mode de formation des stéréotypes visuels va connaître une profonde mutation, en même temps que va s'organiser le discours sur les caractères nationaux. D'une part, l'accent sera mis sur les caractères physiques, à peu près inexistants jusque-là, et qui se développeront parallèlement à l'émergence puis à l'évolution des théories raciales, et d'autre part les différentes "personnalités » (repérables aux traits physiques, au costume, aux mœurs, aux habitats, etc.) seront prises en charge par une syntaxe nouvelle procédant de la mise en série systématique, l'objectif étant de constituer un tout à travers la somme des parties, selon une opération inclusive 
pouvant jouer à plusieurs niveaux ${ }^{13}$. Ainsi, pour la France, des personnalités régionales qui, prises ensembles, constituent la nation: d'où la prolifération d'éditions des «séries de provinces de France » où chaque élément ne vaut que par son inclusion dans la série, c'est-à-dire son identification. Mais en même temps, la personnalité régionale, «bretonne » mettons, est elle-même la résultante d'une série de personnalités locales (" pays » Pagan, Rouzig, Bigouden, etc.) qui la qualifient ${ }^{14}$. Il n'en ira pas différemment du regard porté sur les Indiens, qui évoluera lui aussi d'un système de stéréotypes fondés sur une concaténation d'attributs à fort rendement symbolique pouvant entrer dans un jeu d'échanges relativement large (un régime allégorique), à un autre système de stéréotypes contraints par une double opération d'identification et de qualification (un régime taxinomique). L'intérêt du musée de Catlin, nous l'avons dit plus haut, est que sa présentation se situe précisément à la phase inaugurale de ce basculement, ce qui explique sans doute en partie les problèmes qu'il a pu rencontrer dans son entreprise, ce qui explique aussi pourquoi L'Illustration lui fit un meilleur accueil que l'imagerie. On ne note pas de transition brutale d'un système à l'autre ; il s'agit plutôt d'un changement de point de vue dans la manière commune de percevoir la valeur d'une image, c'est-à-dire en premier lieu sa place et son importance dans l'ensemble des images accessibles à un moment donné. L'imagerie s'attardera dans l'ancien système longtemps après que le nouveau aura pris naissance : les "Incas » à plumes d'autruche auront encore droit de cité, bien qu'ils soient de moins en moins présents et que, dans le système taxinomique, ils soient désormais connotés comme «Indiens de fantaisie ». Réciproquement, les saisies documentaires opérées pour les besoins de la mise en place des nouveaux cadres pourront, dans une certaine mesure, être employées selon les anciens critères, par exemple lorsque l'Amérique des quatre (ou désormais des cinq) Parties du monde, sera figurée par un Sioux; un tel système implique forcément le recours à la synecdoque, puisqu'il faut bien trouver un moyen de représenter les catégories génériques (l'Indien en général, ou l'Amérique). L'imagerie, surtout celle d'Épinal, devra, pour survivre, composer avec ces différents systèmes, intégrant au passage des genres qu'elle avait jusque-là délaissés, comme la caricature ou l'image documentaire. 
Indiens Peaux-Rouges, lithographie coloriée au pochoir, Épinal, Pellerin.

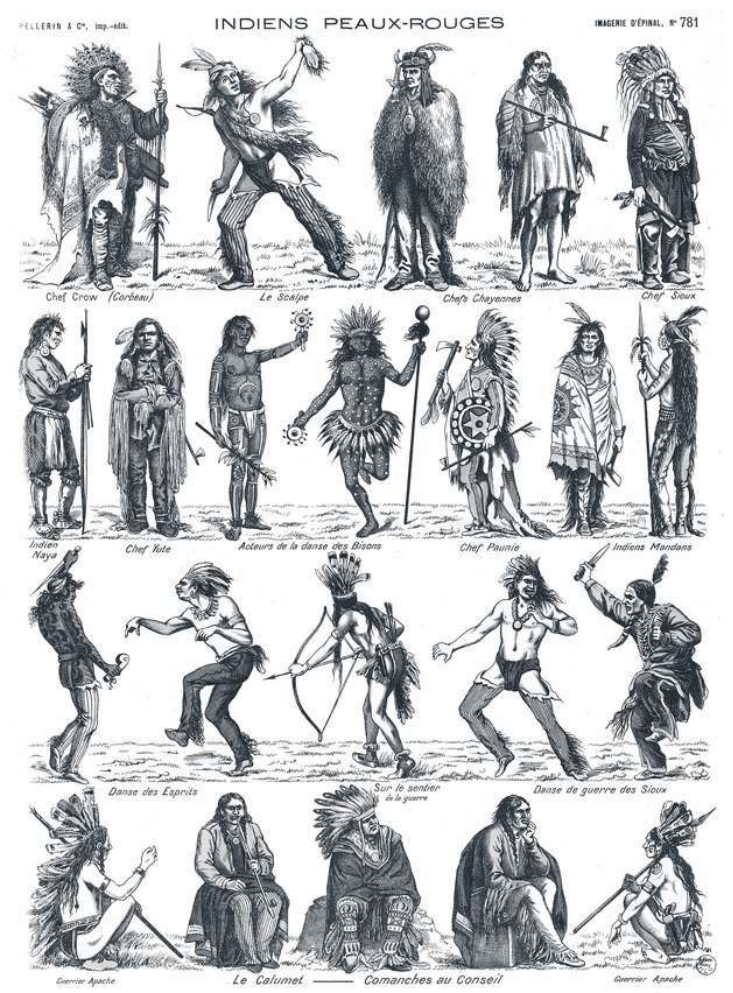

Iconothèque, musée des Civilisations d'Europe et de la Méditerrannée.

Sur ce dernier point - l'arraisonnement de la logique documentaire par l'imagerie d'Épinal - la présentation des Iowas à Paris constitue un événement. Alors que, comme on l'a vu, leur présence avait été complètement ignorée par l'imagerie parisienne, Pellerin crée la surprise en publiant, au début de l'année 1846, une planche intitulée Les Indiens ioways des Montagnes Rocheuses (planche XIV). Elle est signée Jean-Baptiste Vançon, un graveur spinalien de la jeune génération (il a alors vingt-cinq ans), très doué, qui gravera pour Pellerin une centaine de bois; les Ioways, qui figurent au dépôt légal le 20 février 1846, sont sa dixième planche. Si l'on a en tête la production classique d'Épinal, tout est nouveau dans cette estampe: la disposition, le thème, le traitement et les sources. L'image se compose d'un bandeau à la partie supérieure, représentant la rencontre entre les Iowas et la famille royale, surmontant neuf vignettes figurant des scènes indiennes. Le principe de la disposition en bandeau est récent, et tout à fait exceptionnel dans l'image d'Épinal, de même d'ailleurs que les vignettes horizontales groupées par séries de trois. La combinaison des deux constitue une composition unique, et une image extrêmement réfléchie, car cette disposition atypique a une visée précise de renforcement du contenu des vignettes et d'homogénéisation de la planche. C'est la première fois que Pellerin publie une image composée d'une accroche événementielle, et pas n'importe laquelle puisqu'il ne s'agit de rien moins que d'une ambassade royale, qui introduit un ensemble documentaire à prétention réaliste ; en bref, un reportage en images au sens moderne du terme. La formule en elle-même est une nouveauté absolue ; certes elle se rapproche de celle des journaux illustrés, mais pas de L'Illustration ni du Magasin pittoresque, qui accordent une large place au texte. Elle annonce plutôt ce que seront, dans la seconde moitié du siècle, les publications dites de «presse populaire illustrée » comme le Journal 
illustré, la Presse illustrée, le Quotidien illustré, etc., journaux qui, en 1846, n'ont pas encore vu le jour. À cet égard, Les Indiens ioways des Montagnes Rocheuses constitue une énigme. Deuxième mystère, non résolu : l'auteur. Seul Vançon a signé, pourtant il ne dessinait pas, mais interprétait dans une technique de bois de fil extrêmement subtile, beaucoup plus élaborée à maints égards que celle des graveurs de L'illustration, des compositions réalisées par d'autres. Or, les dessins originaux n'ont pas été retrouvés ; sans doute ont-ils été, comme une grande partie des archives et des anciens bois de Pellerin, détruits dans l'incendie qui ravagea la fabrique en 1888. L'identité du dessinateur restera donc un mystère ; en revanche, on peut assez facilement reconstituer la genèse de la planche. On se souvient qu'en 1827 , alors que d'autres centres imagiers rendaient compte de la visite des Osages, Pellerin était resté en retrait. En retrait mais pas inactif puisqu'il avait édité, l'année suivante, les Sauvages de l'Amérique. Sans doute comptait-il, avec raison, sur la vague d'intérêt provoquée par les Osages pour faciliter la vente d'une planche à sujet indien. Mais, par manque de documentation, il avait été contraint de se rabattre sur une représentation de l'Indien classique des allégories. En 1845, la situation de la firme spinalienne a notablement évolué, passant de l'atelier artisanal à une forme protoindustrielle; les circuits de diffusion se sont élargis, et les contacts sont beaucoup plus aisés avec les sources de documentation parisiennes. Les Sauvages de l'Amérique, même s'ils avaient fait l'objet de rééditions ultérieures (au moins une en 1841) avaient constitué un loupé pour la firme spinalienne; aussi Pellerin a-t-il mandaté un de ses contacts parisiens pour rapporter à Épinal toute la documentation disponible sur les Iowas.

On peut, à partir de l'examen de la planche, se faire une idée assez précise de la méthode employée. Le dessinateur était un bon connaisseur des Salons parisiens, il avait une culture visuelle importante, et par ailleurs il avait vu le spectacle de la salle Valentino et le Musée Catlin. Outre le grand dessin représentant la rencontre entre le roi et les Iowas, il a ramené trois séries de dessins : un reportage direct du spectacle, pris sur le vif, des copies de certaines œuvres exposées par Catlin et une documentation complémentaire sur divers aspects des mœurs des Indiens, à partir de sources extérieures à l'œuvre de Catlin.

15 Le premier registre de vignettes représente des éléments du spectacle, légendés de manière assez élémentaire : «Danse du scalp. Célébration d'une victoire. Cette cérémonie dure pendant quinze nuits consécutives ", «Danse de la recherche. Ils font le geste de découvrir la trace du gibier ou de l'ennemi », et «Musique des Indiens». Pellerin et son mandataire ont eu entre les mains la notice du spectacle, celle dont nous parlions plus haut, qui comporte les bois de Porret. On y trouve la danse du scalp, dont les premières phrases de la notice inspirent directement celle d'Épinal : «Par cette scène barbare et animée, les Indiens célèbrent une victoire; elle est exécutée pendant quinze nuits consécutives, lorsqu'une troupe de guerriers revient d'une expédition avec les scalps qu'elle a arrachés aux têtes de ses ennemis. » La représentation gravée par Vançon est sans doute beaucoup plus proche de ce qu'ont pu voir les spectateurs que la version donnée par Porret, qui reprend une peinture de Catlin publiée sous forme lithographiée dans The North American Indian Portfolio (Londres 1844, New York 1845). La Danse de la recherche correspond pour sa part à ce que la notice nomme La danse de l'approche. Le témoignage direct ne fait aucun doute, il n'est que de comparer avec le tableau de Karl Girardet pour reconnaître qu'on a bien affaire au même spectacle. On a donc là le seul témoignage direct du spectacle qui soit destiné à un très large public, puisque l'image, qui 
fait l'objet d'un premier tirage à 5000 exemplaires, sera dans les hottes des colporteurs et sur les étals des vendeurs d'images à la feuille dès le printemps 1846 .

Le second registre reproduit, avec une certaine liberté, trois peintures de Catlin. Celle de gauche, la plus connue, est légendée de la façon suivante: Chasse aux buffles. Les Indiens s'approchent d'un troupeau de buffles sous des peaux de loups blancs. C'est la seule représentation que l'on retrouve pratiquement à l'identique dans L'Illustration (où elle était intitulée Chasse aux buffles par surprise) et sur l'image de Pellerin. Celle de droite, Chasse au bison, reprend une autre peinture célèbre de Catlin, également très reproduite en lithographie. Curieusement, alors que toutes les versions connues montrent un cavalier tirant à l'arc, sur l'estampe d'Épinal, l'arc est remplacé par une lance, même si le «bison» porte une flèche plantée dans l'échine, telle une banderille de corrida. La vignette centrale reprend, avec beaucoup plus de libertés, une peinture moins connue et moins reproduite, intitulée Attacking The Grizzly Bear.

17 Le troisième registre, plus difficile à analyser, est composé de trois scènes ainsi légendées: "Troupe d'Indiens en marche. Les tentes sont roulées et les perches servent de voitures ", "Sépulture des Indiens, les femmes présentent des plats de viande aux squelettes de leurs parents» et «Médecin sorcier visitant un malade et conjurant la maladie ». Il ne s'agit pas, cette fois, de reproductions, ou même d'interprétations, mais de compositions ad hoc, même si certains motifs sont inspirés par des œuvres plus ou moins connues (ainsi, l'intérieur du wigwam de la vignette de gauche est imité d'une aquarelle de Peter Rindisbacher ${ }^{15}$. On peut admirer le talent de documentaliste du correspondant de Pellerin car en 1845 de telles scènes, qui sont devenues depuis des lieux communs à travers les films ou les bandes dessinées, sont très difficiles à se procurer. Où, par exemple, trouver la coutume funéraire dépeinte sur la vignette centrale ? On trouve certes un tableau de Karl Bodmer, intitulé Funeral Scaffold of the Sioux Chief, publié en aquatinte dans Travels in the Interior of North America1839-1843. On trouve également, exposé au Salon de 1806, une toile de Louis Hersent intitulée Le Tombeau aérien, coutume américaine, mais rien qui s'approche de l'image exacte gravée par Vançon. De même, L'Illustration a publié le 24 mai 1845 une gravure intitulée Émigration des Peaux-Rouges, qui montre la technique des perches représentée dans la première vignette, mais là encore il s'agit d'une influence fort lointaine. 


\section{SAUVAGES DE L'AMERIQUE.}
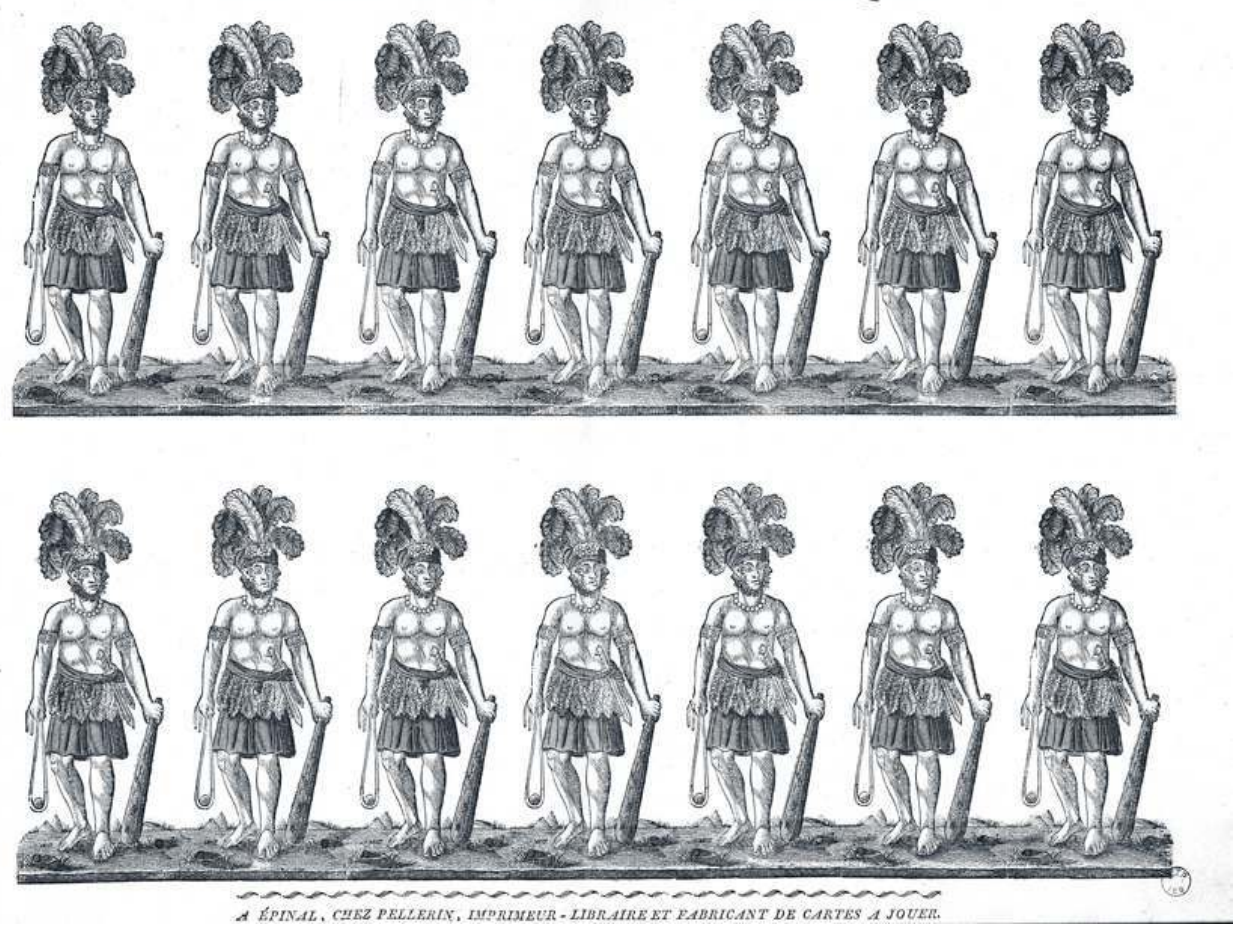

Iconothèque, musée des Civilisations d'Europe et de la Méditerrannée.

Suivant la coutume de l'imagerie d'Épinal, l'origine des scènes n'est absolument pas mentionnée. Et même dans cette planche, qui met en avant la valeur documentaire, le travail du stéréotype est à l'œuvre, tant au niveau des thèmes qu'au niveau des motifs. Ainsi, les Indiens « Ioways » représentent l'ensemble des Indiens des Plaines : faire figurer une coutume funéraire sioux ne pose aucun problème. En ce qui concerne les motifs, il faut porter attention aux coiffures, qui sont du type « osages-iowas » décrit plus haut ; or, sur les peintures de Catlin, tant la chasse au buffle que la chasse au grizzli mettent en scène des Indiens à la chevelure abondante.

Ce qui se donne comme témoignage direct est donc largement le résultat d'une opération de recomposition. Cela est particulièrement visible au niveau du bandeau supérieur, qui représente l'entrevue avec la famille royale. Contrairement à Girardet, l'envoyé de Pellerin n'a pas assisté à l'entrevue. Celle-ci est en revanche fort bien décrite dans la notice et elle se présente en deux temps : la réception officielle et le spectacle; l'artiste d'Épinal choisit de traiter la première, sur le modèle des ambassades. Or, la notice donne l'impression d'une réception beaucoup plus chaleureuse, en tout cas moins protocolaire : «Après s'être fait présenter les chefs et les guerriers selon le rang de chacun, le roi causa longuement des mœurs et des habitudes des Sauvages, disant que lui-même, dans sa jeunesse, avait visité un grand nombre d'Indiens américains dans leurs propres wigwams sur les rives de l'Ohio et du Mississippi. Sa Majesté donna ensuite, de sa main, une médaille d'or à chaque chef et une médaille d'argent à chaque guerrier... » La vignette représente le moment où Louis-Philippe donne la médaille au Nuage-Blanc; mais comment représenter une telle scène à partir d'un texte? Comment imaginer la disposition des lieux, les personnages présents? Pour les Indiens, la chose est simple 
puisque l'auteur du dessin les a vus de près ; la légende de la vignette suit à la lettre le premier texte de la notice qui donne les noms des Iowas, et le dessin est agencé pour qu'on puisse reconnaître aisément chacun d'entre eux. Mais comment procéder en ce qui concerne la famille royale, que la légende, d'ailleurs, ne détaille pas? On reconnaît, derrière le roi, la reine Marie-Amélie, ainsi que la princesse Marie-Adélaïde, entre les deux femmes se tient un enfant qui ressemble au duc de Nemours et à l'extrême gauche un jeune homme qui pourrait être le duc d'Orléans, c'est-à-dire les deux fils aînés de Louis-Philippe... tels qu'ils étaient en 1830; rappelons pour l'anecdote que le duc d'Orléans est mort dans un accident en 1842 et que le duc de Nemours a 31 ans en 1845. On pourrait sans doute rechercher lesquels des fils et petits-fils du roi pouvaient raisonnablement correspondre aux deux personnages représentés, mais il est plus simple de suivre la logique de réemploi que nous avons remarquée à propos du tableau de Rindisbacher. On observe alors que la partie gauche de la vignette est composée sur le modèle d'un tableau de François-Joseph Heim, peint en 1837 et intitulée Le duc d'Orléans reçoit au Palais-Royal la Chambre des pairs, 7 août 1830 qui présente sur la gauche, dans la même position, Ferdinand, alors âgé de vingt ans.

On ne sait pas si l'estampe fit l'objet de retirages, sa carrière ne dura que deux ans, car il n'était évidemment plus question de la produire après février 1848 , et les bois des scènes indiennes ne semblent pas avoir été réemployés. Malgré tout, par son mode de diffusion, par le traitement qu'elle donne de l'apparence physique des Iowas, très proche de celle, déjà ancrée, des Osages, par le caractère frappant des scènes de danse et de chasse, elle contribua sans doute plus au renouvellement de l'image de l'Indien auprès du grand public que ne le firent les numéros de L'Illustration. Il est bien sûr hasardeux d'estimer la réception d'une telle image en l'absence de données directes, mais on peut tabler sur deux facteurs. Tout d'abord elle est parfaitement cohérente avec l'horizon d'attente ${ }^{16} \mathrm{du}$ public de l'imagerie, puisque les Iowas d'Épinal ne contredisent pas les représentations antérieures de type allégorique, ils les décalent en les précisant. Ensuite, deux scènes s'inscriront durablement dans les avatars des représentations indiennes destinées au grand public : la danse et la chasse au bison.

21 Ce n'est que bien plus tard qu'une autre image entièrement consacrée aux Indiens est éditée par Pellerin, en 1891, soit deux ans après la première tournée de Buffalo Bill en Europe. Intitulée Indiens Peaux-Rouges, elle présente des Sioux, des Cheyennes, un chef crow, des Comanches et des Apaches. L'époque a changé et l'on se situe dans le cadre, décrit plus haut, d'un stéréotype qui fonctionne en régime taxinomique. Là encore, il est aisé de retrouver des influences, pour cette image qui se compose comme un répertoire de figures; par exemple les deux "guerriers apaches» abondamment munis de plumes figurant de part et d'autre du registre du bas sont recopiés très précisément d'une gravure de L'Illustration de 1889 présentant le spectacle de Buffalo Bill. Mais au milieu de ces Indiens, qui font partie soit de la troupe de Buffalo Bill, soit des « zoos humains » du Jardin d'Acclimatation, on remarque, au milieu du deuxième registre, deux personnages nommés "Acteurs de la danse des bisons ». Il s'agit de deux Mandans figurant au centre du tableau de Catlin qui avait déjà été reproduit dans L'Illustration du 7 juin 1845. Comment et pourquoi ils sont arrivés sur l'estampe de 1891 est une question à laquelle il est à peu près impossible de répondre. En revanche, on peut repérer quelques réemplois de ce tableau ou du motif des deux danseurs: sur une chromolithographie publicitaire pour la marque Liebig, éditée en 1911, par exemple. 
Dans les années 1960, la chocolaterie COOP édite une série d'images publicitaires intitulée Sur la piste des Indiens ; 28 images de cette série qui en compte 90 sont tirées des œuvres de Catlin. Parallèlement, la firme publie un album collector, comme c'était l'habitude à l'époque; il s'agit d'un livre de 64 pages à prétention encyclopédique où Catlin bénéficie d'une notice biographique accompagnée de son autoportrait. Les textes, qui présentent l'histoire des Indiens des États-Unis, sont pour certains très fortement inspirés des écrits du peintre; enfin, plusieurs encadrés sont explicitement identifiés par la mention "d'après Catlin ». Ainsi, plus d'un siècle après sa visite à Paris, son œuvre demeure une référence pour la représentation de l'Indien, référence sporadique mais fortement ancrée, comme en témoigne la présence du danseur mandan sur la couverture de L'Origine des manières de table de Claude Lévi-Strauss, publié chez Plon en 1968.

\section{BIBLIOGRAPHIE}

BERTHO, Catherine

1980 « L'invention de la Bretagne, genèse sociale d'un stéréotype ", Actes de la recherche en sciences sociales 35. Paris, Minuit : 45-62.

GASNAULT, François

1986 Guinguettes et lorettes. Bals publics et danse sociale à Paris entre 1830 et 1870. Paris, Aubier.

HONOUR, Hugh, Ed.

1976 L'Amérique vue par l'Europe, Galeries nationales du Grand Palais. Paris, RMN.

JAUSS, Hans Robert

1978 (1974) Pour une esthétique de la réception. Paris, Gallimard.

MAGUET, Frederic

1994 « De la série éditoriale dans l'imagerie. L'exemple des costumes régionaux », Ethnologie

française 2. Paris, Armand Colin : 226-242.

SAND, George

2004 (1845-46) Le Diable à Paris, recueil. Paris, Mille et une nuits, Arthème-Fayard.

THIESSE, Anne-Marie

1999 La Création des identités nationales. Europe XVIII ${ }^{e}-X X^{e}$ siècle. Paris, Seuil.

WIESINGER, Veronique, éd.

1992 Sur le sentier de la découverte. Rencontres indiennes du XVI ${ }^{e}$ au XX ${ }^{e}$ siècle, Musée national de la coopération franco-américaine, Château de Blérancourt. Paris, RMN.

Musée Goya

1992 La France et la conquête de l'Amérique. Castres, Albaron. 


\section{NOTES}

1. À ses débuts, L'Illustration tire à 13500 exemplaires, pour atteindre 35000 exemplaires en 1848 . On est bien sûr très loin des tirages à 300000 que connaîtra le journal durant la Première Guerre mondiale, mais en 1845, un tel chiffre est considérable. Il faut rappeler qu'à cette époque l'image est rare et coûte cher et, dans sa catégorie, L'Illustration restera sans concurrent sérieux jusqu'en 1857, lorsque paraîtra Le Monde illustré. Seuls préexistent, mais dans un genre différent puisqu'il s'agit de la presse d'éducation, Le Magasin pittoresque et Le Musée des familles (qui datent tous deux de 1833).

2. Johannot a, quelques années auparavant, illustré les œuvres complètes de Fennimore Cooper.

3. Peintre reconnu au Salon pour ses sujets exotiques, collaborateur régulier, et sur une longue période, de L'Illustration et du Magasin pittoresque, il avait, comme de nombreux artistes de l'époque, beaucoup voyagé (en Espagne, en Afrique du Nord, au Mexique, en Russie, au Caucase). On lui doit, en 1832, la majorité des dessins du Voyage pittoresque en Espagne, au Portugal et sur la côte d'Afrique de Tanger à Tétouan du baron Taylor.

4. Le texte complet est présenté dans la réédition de 2004.

5. BNF Estampes, Za 198 pet. In-folio, reproduites dans le catalogue de l'exposition Sur le sentier de la découverte 1992.

6. Je tiens à remercier Jacques Cheyronnaud, chercheur au SHADYC, de m'avoir donné ces précisions et de m'avoir indiqué le travail de François Gasnault.

7. Musée national de la coopération franco-américaine de Blérancourt, reproduites dans le catalogue de l'exposition Sur le sentier de la découverte 1992.

8. Cette différenciation se lit très bien sur le plan économique, l'imagerie et les journaux illustrés ne s'adressent pas aux mêmes publics: L'Illustration coûte 75 centimes le numéro, et se vend surtout par abonnement; on est loin de l'image à deux sous d'Épinal ou de la lithographie, sensiblement au même tarif, de la veuve Turgis.

9. Par exemple chez Bulla, vers 1845, qui édite une lithographie de Lermercier d'après un dessin de Chasselat intitulée Atala et Chactas secourus par le père Aubry; le modelé des corps est tout à fait néoclassique, Chactas est vêtu d'une toge et d'une cape et coiffé d'un bandeau surmonté de trois larges plumes ; Atala, pour sa part, est figurée comme une jeune Européenne.

10. Buste en plâtre représentant Esprit-Noir, exposé au Salon de 1827, musée Carnavalet, reproduit dans le catalogue de l'exposition Sur le sentier de la découverte 1992.

11. Par exemple sur la gravure de Grasset Saint-Sauveur Homme et femme Ottawas de l'édition de 1806 des Voyages.

12. Voir le catalogue de l'exposition La France et la conquête de l'Amérique 1992.

13. Sur cette construction, voir Bertho-Lavenir 1980 et Thiesse 1999.

14. Je renvoie ici à un travail antérieur, Maguet 1994.

15. Aquarelle datant de 1829-1834 conservée au West Point Museum qui a été présentée à l'exposition L'Amérique vue par l'Europe (1976) sous le numéro 294 (reproduite dans le catalogue).

16. Toute notre analyse de la réception populaire de l'image doit beaucoup aux travaux qu'Hans Robert Jauss a conduits dans le domaine de l'histoire littéraire. Les principaux concepts de l'École de Constance (horizon d'attente fondé sur une connaissance préalable d'un genre donné ainsi que sur des formes et des thématiques préexistantes, écart esthétique, effet) sont parfaitement applicables dans le domaine visuel dans un cas comme celui qui nous intéresse ici (Jauss 1974). 


\section{RÉSUMÉS}

La présentation, en 1845, du musée itinérant de George Catlin et du spectacle donné par les Iowas sont immédiatement relayés par le magazine L'Illustration, puis, avec quelque délai, par l'imagerie d'Épinal. En un siècle qui connaît l'avènement de l'image pour tous, ces deux vecteurs entraînent un bouleversement total de la représentation des Amérindiens au sein du public français. Déjà entamé avec la visite des Osages en 1827, le processus consiste en une mutation profonde du mode de formation des stéréotypes visuels. Une image à prétention réaliste, puisque incluant des traits réellement observés, va entrer dans un jeu de coexistence et de substitution avec l'image allégorique classique du "sauvage d'Amérique». Se donnant comme un témoignage direct, l'imagerie de l'Indien d'après Catlin résulte en fait d'une opération de recomposition de traits, de motifs et de situations ayant pour effet de produire une représentation assimilable par le public, encore largement active aujourd'hui.

In 1845, the presentation of George Catlin's travelling museum and the accompanying show put on by the Iowa Indians were immediately depicted in "L'Illustration" magazine and, not long after, in Épinal prints. In a century where pictorial images were becoming available to all, these two mediums led to a complete turnaround in the way that the French public pictured Amerindians. The process had already begun in 1827 with the visit of the Osage Indians, and consisted in a deep-seated change in the way that visual stereotypes came to be formed. An image laying claims to realism, incorporating actually observed characteristics, began to coexist with then replace for the classic allegorical image of the "American savage". Presented as eyewitness evidence, Catlin's depiction of the Indian in fact resulted from a reconstruction of characteristics, motifs and situations, and gave rise to a representation easily assimilated by the public, and which still has wide currency today.

\section{INDEX}

Mots-clés : Amérindien, image, imagerie populaire, stéréotypes visuels, XIXe siècle Keywords : American Indian, nineteenth century, popular imagery, visual stereotypes

\section{AUTEUR}

\section{FRÉDÉRIC MAGUET}

Responsable de l'iconothèque du musée des Civilisations de l'Europe et de la Méditerranée, frederic.maguet@culture.gouv.fr 